\title{
On Signed Domination of Grid Graph
}

\author{
Mohammad Hassan, Muhsin Al Hassan, Mazen Mostafa \\ Department of Mathematics, Faculty of Science, Tishreen University, Lattakia, Syria \\ Email: sf-qau@tishreen.edu.sy, mmuuhhssiinn@gmail.com, mazenmostafa1979@gmail.com
}

How to cite this paper: Hassan, M., Al Hassan, M. and Mostafa, M. (2020) On Signed Domination of Grid Graph. Open Journal of Discrete Mathematics, 10, 96-112. https://doi.org/10.4236/ojdm.2020.104010

Received: August 9, 2020

Accepted: October 24, 2020

Published: October 27, 2020

Copyright (c) 2020 by author(s) and Scientific Research Publishing Inc. This work is licensed under the Creative Commons Attribution International License (CC BY 4.0).

http://creativecommons.org/licenses/by/4.0/

\begin{abstract}
Let $G(V, E)$ be a finite connected simple graph with vertex set $V(G)$. A function $f: V(G) \rightarrow\{-1,1\}$ is a signed dominating function if for every vertex $V$ $\in V(G)$, the sum of closed neighborhood weights of $v$ is greater or equal to 1 . The signed domination number $\gamma_{s}(G)$ of $G$ is the minimum weight of a signed dominating function on $G$. In this paper, we calculate the signed domination numbers of the Cartesian product of two paths $P_{m}$ and $P_{n}$ for $m=6$, 7 and arbitrary $n$.
\end{abstract}

\section{Keywords}

Grid Graph, Cartesian Product, Signed Dominating Function, Signed

Domination Number

\section{Introduction}

Let $G$ be a finite simple connected graph with vertex set $V(G)$. The neighborhood of $v$, denoted $N(v)$, is set $\{u: u v \in E(G)\}$ and the closed neighborhood of $v$, denoted $N[v]$, is set $N(v) \cup\{v\}$. The function $f$ is a signed dominating function if for every vertex $v \in V$, the closed neighborhood of $v$ contains more vertices with function value 1 than with -1 . The weight of $f$ is the sum of the values of $f$ at every vertex of $G$. The signed domination number of $G, \gamma_{s}(G)$, is the minimum weight of a signed dominating function on $G$.

In [1] [2] [3] [4], Dunbar et al. introduced this concept, in [5] Haas and Wexler had found the signed domination number of $P_{2} \times P_{n}$ and $P_{2} \times C_{n}$. In [6] Hosseini gave a lower and upper bound for the signed domination number for any graph. In [7] Hassan, Al Hassan and Mostafa had found the signed domination number of $P_{m} \times P_{n}$ for $m=3,4,5$ and arbitrary $n$.

We consider when we represent the $P_{m} \times P_{n}$ graph. The weight of the black circle is 1 , and the white circles refer to the graph vertices which weight -1 .

Let $f$ be a signed dominating function of the $P_{m} \times P_{n}$ and $A=\{v \in V: f(v)=1\}$, 
$V=\{v \in V: f(v)=-1\}$, then $\gamma_{s}\left(P_{m} \times P_{n}\right)=m \cdot n-2|B|=|A|-|B|$. Let $K_{j}$ be the $f^{\text {th }}$ column vertices, and also $A_{j}=\left\{v \in K_{j}: f(v)=1\right\}, B_{j}=\left\{v \in K_{j}: f(v)=-1\right\}$.

\section{Main Results}

In this paper we will show tow theorem to find the signed domination number of Cartesian product of $P_{m} \times P_{n}$.

Theorem 2.1. For $n \geq 1$ then

$$
\gamma_{s}\left(P_{6} \times P_{n}\right)=\left\{\begin{array}{l}
2 n ; \text { If } n \equiv 1(\bmod 5), \\
2 n+2 ; \text { If } n \equiv 2(\bmod 5), \\
2 n+4 ; \text { If } n \equiv 0,3,4(\bmod 5) .
\end{array}\right.
$$

Proof:

Let $f$ be a signed dominating function of $\left(P_{6} \times P_{n}\right)$, then for any $j$ were $2 \leq j \leq$ $n-3$, then $\sum_{k=j-1}^{j+2}\left|B_{K}\right| \leq 8$. We discuss the following cases:

Case a. $\left|B_{j}\right|=4$ :

we notice that the first and last columns can't include four of the $B$ set vertices, but in the case $2 \leq j \leq n-3$ and $\left|B_{j}\right|=4$, then the vertices $(1, j),(3, j),(4$, $j),(6, j) \in B$, and all of the $j-1^{\text {th }}, j+1^{\text {th }}$ column's vertices don't contain any one of the B set vertices, so the $(1, j+2),(6, j+2)$ vertices, then the $j+2^{\text {th }}$ column includes three of the $B$ set vertices at most (Figure 1 ).

Case b. $\left|B_{j}\right|=3$ :

We discuss the following cases:

b-1. If $(1, j),(3, j),(4, j) \in B$ then both of the $j-1^{\text {th }}, j+1^{\text {th }}$ columns include at most one of the $B$ set vertices, then the $j+2^{\text {th }}$ column includes at most three of the $B$ set vertices.

b-2. If $(1, j),(3, j),(5, j) \in B$ then the $j-1^{\text {th }}$ and $j+1^{\text {th }}$ columns include at most two of the $B$ set vertices, and the $j+1^{\text {th }}$ column includes three of the $B$ set vertices.

b-3. If $(1, j),(3, j),(6, j) \in B$ then both of the $j-1^{\text {th }}, j+1^{\text {th }}$ columns include at most one of the $B$ set vertices. And the $j+2^{\text {th }}$ column includes two of the $B$ set vertices.

b-4. If $(1, j),(4, j),(5, j) \in B$ then only one of the $j-1^{\text {th }}, j+1^{\text {th }}$ columns include at most one of the $B$ set vertices, so $(1, j+2) \in A$, then the $j+2^{\text {th }}$ column includes at most three of the $B$ set vertices.

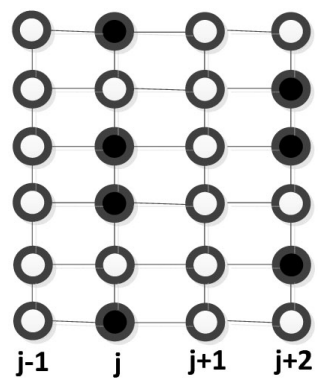

Figure 1. Case a. 
b-5. If $(1, j),(4, j),(6, j) \in B$ then both of the $j-1^{\text {th }}, j+1^{\text {th }}$ columns include at most one of the $B$ set vertices. Also $(1, j+2),(4, j+2)$ and $(6, j+2) \in A$ then only two of the $j+2^{\text {th }}$ vertices belong to $B$ set.

b- 6 . If $(2, j),(3, j),(6, j) \in B$ then only one of the $j-1^{\text {th }}, j+1^{\text {th }}$ column's vertices belong to the $B$ set vertices, then the $j+2^{\text {th }}$ column include at most four of the $B$ set vertices (Figure 2).

Case c. $\left|B_{j}\right|=2$ :

We discuss the following cases:

c-1. If $(1, j),(3, j) \in B$ then all of the $j-1^{\text {th }}, j+1^{\text {th }}, j+2^{\text {th }}$ columns include at most two of the $B$ set vertices (Figure 3 ).

c-2. If $(1, j),(4, j) \in B$ and the $j-1^{\text {th }}$ column include two of the $B$ set vertices then the $j+1^{\text {th }}$ column include at most one of the $B$ set vertices, so the $j+2^{\text {th }}$ column include at most three vertices (Figure 4 ).

c-3. If $(1, j),(5, j) \in B$ or $(1, j),(6, j) \in B$, then all of the $j-1^{\text {th }}, j+1^{\text {th }}, j+2^{\text {th }}$ columns include at most two of the $B$ set vertices (Figure 5).

c-4. If $(2, j),(3, j) \in B$ then if the $j-1^{\text {th }}$ column includes two of the $B$ set vertices, then the $j+1^{\text {th }}$ column includes at most one of the $B$ set vertices, so the $j+$ $2^{\text {th }}$ column includes at most three vertices (Figure 6).

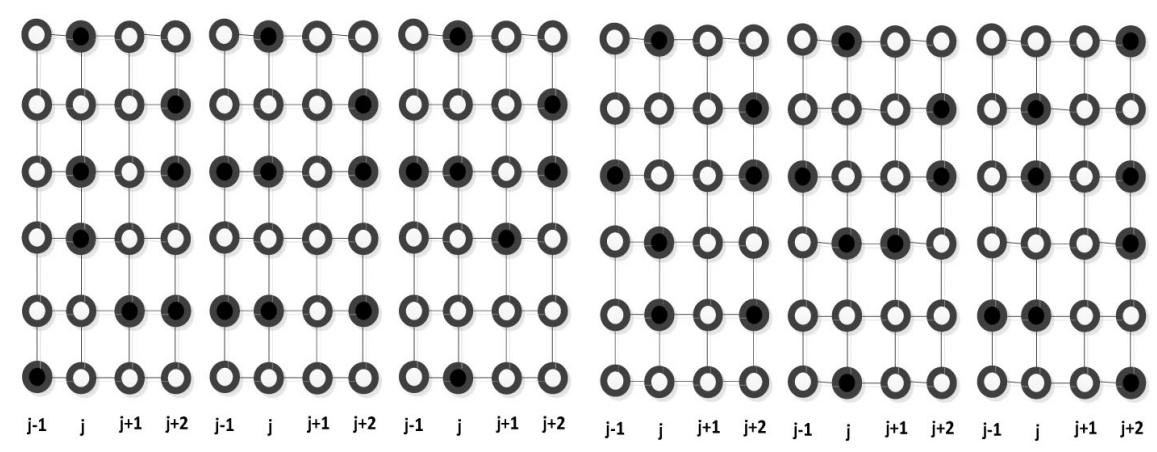

Figure 2. Case b.

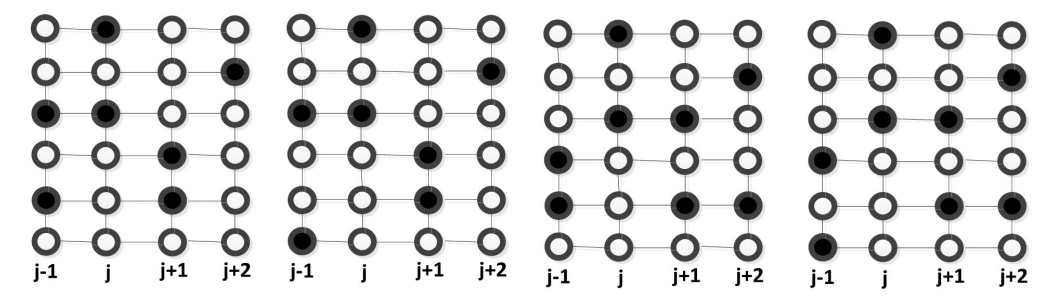

Figure 3. Case c-1.

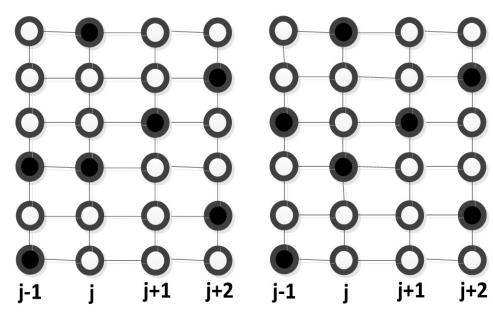

Figure 4. Case c-2. 
c-5. If $(2, j),(4, j) \in B$ then the $j-1^{\text {th }}$ column includes at most three of the $B$ set vertices, it is $(2, j-1),(4, j-1),(6, j-1) \in B$, so the $j+1^{\text {th }}$ column includes one of the B set vertices, also the $j+2^{\text {th }}$ column includes three of the $B$ set vertices and both of the $j-2^{\text {th }}, j+3^{\text {th }}$ columns don't include any one of the $B$ set vertices, so the $j+4^{\text {th }}$ column includes four of the $B$ set vertices and the $j-3^{\text {th }}$ column includes three of the $B$ set vertices. then the eight columns include sixteen of the B set vertices. In other cases stay $\sum_{k=j-1}^{j+2}\left|B_{K}\right| \leq 8 \quad$ (Figure 7).

c-6. If $(2, j),(5, j) \in B$ then all of the $j-1^{\text {th }}, j+1^{\text {th }}, j+2^{\text {th }}$ columns include at most two of the $B$ set vertices (Figure 8 ).

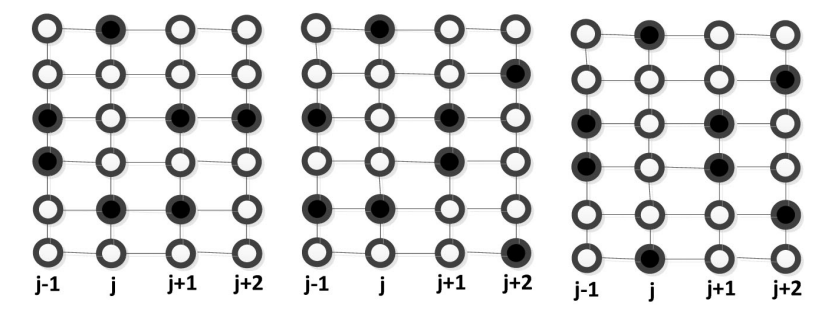

Figure 5. Case c-3.

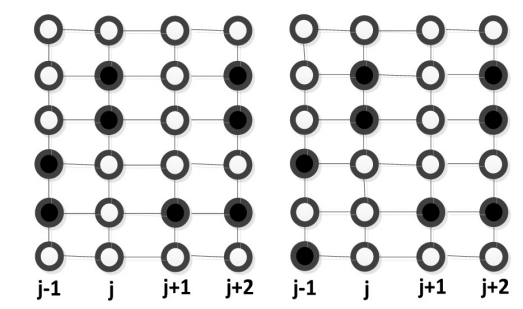

Figure 6. Case c-4.

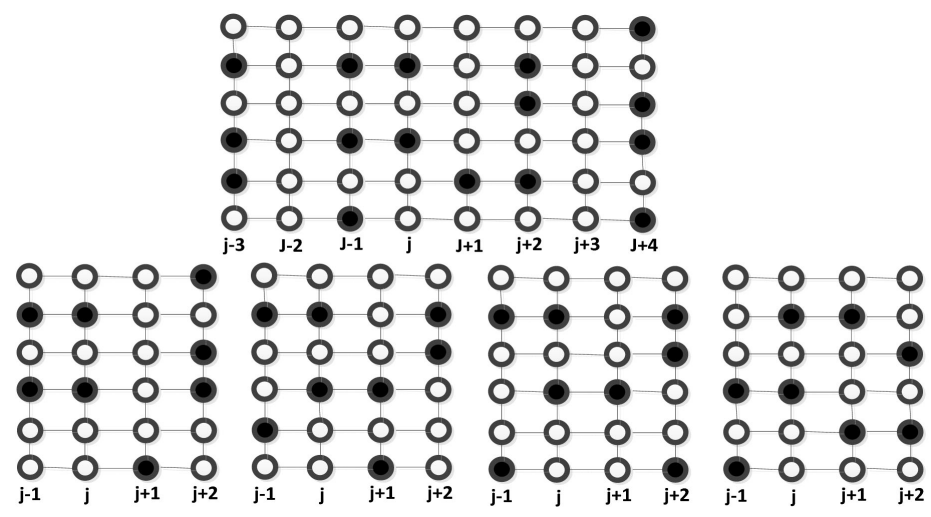

Figure 7. Case c-5.

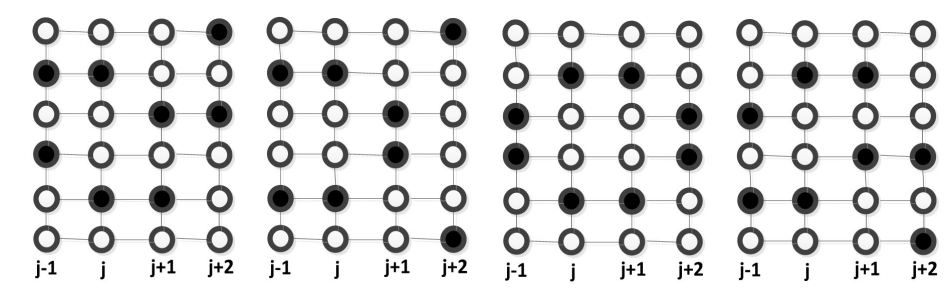

Figure 8. Case c-6. 
c-7. If $(3, j),(4, j) \in B$ then all of the $j-1^{\text {th }}, j+1^{\text {th }}, j+2^{\text {th }}$ columns include at most two of the B set vertices (Figure 9).

Case d. $\left|B_{j}\right|=1$ :

We discuss the following cases:

d-1. If $(1, j) \in B$ or $(3, j) \in B$ or $(4, j) \in B$ or $(6, j) \in B$ then the $j-1^{\text {th }}$ column includes at most three of the $B$ set vertices also both of the $j+1^{\text {th }}, j+2^{\text {th }}$ columns include at most two of the $B$ set vertices (Figure 10).

d-2. If $(2, j) \in B$ or $(5, j) \in B$ then both of the $j-1^{\text {th }}, j+1^{\text {th }}$ columns includes at most three of the $B$ set vertices, and the $j+2^{\text {th }}$ column includes at most one of the $B$ set vertices (Figure 11).

From the previous cases we conclude $\gamma_{s}\left(P_{6} \times P_{n}\right) \geq 2 n$.

To find the upper bound of the signed domination number of $\left(P_{6} \times P_{n}\right)$ graph, let's define (Figure 12).

$$
\begin{aligned}
B=\{ & (1,1+5 i),(6,1+5 i): 0 \leq i \leq\left\lfloor\frac{n-1}{5}\right\rfloor \\
& \cup(3,2+5 i),(4,2+5 i): 0 \leq i \leq\left\lfloor\frac{n+2}{5}\right\rfloor \\
& \cup(2,3+5 i),(5,3+5 i): 0 \leq i \leq\left\lfloor\frac{n+3}{5}\right\rfloor \\
& \cup(2,4+5 i),(5,4+5 i): 0 \leq i \leq\left\lfloor\frac{n+4}{5}\right\rfloor \\
& \left.\cup(3,5+5 i),(4,5+5 i): 0 \leq i \leq\left\lfloor\frac{n+5}{5}\right\rfloor\right\}
\end{aligned}
$$

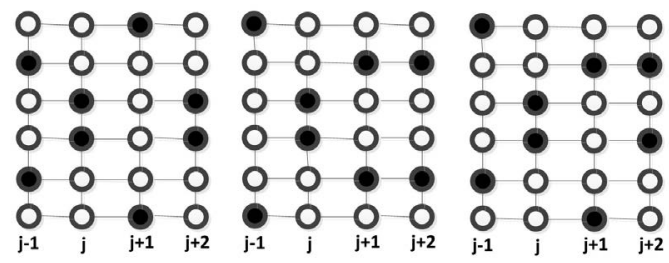

Figure 9. Case c-7.

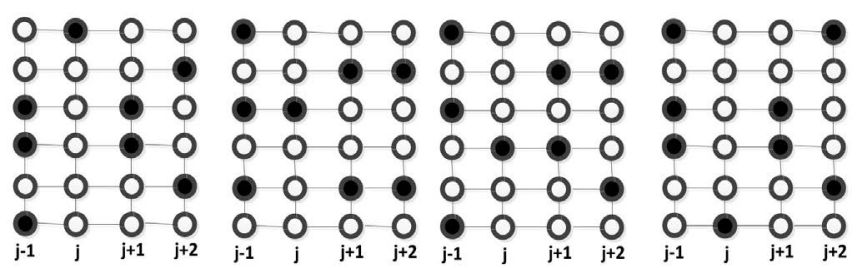

Figure 10. Case d-1.

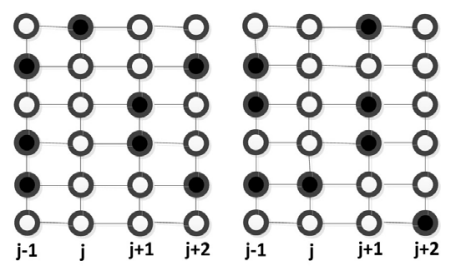

Figure 11. Case d-2. 


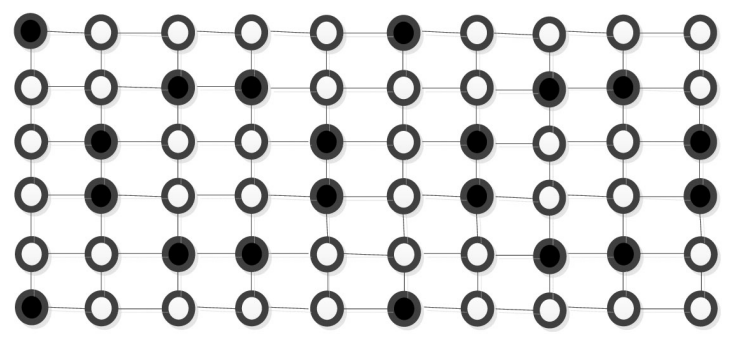

Figure 12. B set.

Case $n \equiv 1(\bmod 5)$.

If $B$ is the previously defined set and represents the vertices have the weight -1 , then every one of the $P_{6} \times P_{n}$ vertices achieves the signed dominating function, and $|B| \geq 2 n$, then: $\gamma_{s}\left(P_{6} \times P_{n}\right) \leq 6 n-2(2 n)=2 n$. Consequently: $\gamma_{s}\left(P_{6} \times P_{n}\right)=2 n: n \equiv 1(\bmod 5)$ (Figure 13$)$.

Case $n \equiv 2(\bmod 5)$.

In this case, we delete one of the two vertices $(3, n)$ or $(4, n)$ from the previously defined set $B$ vertices, then the signed domination number will increase by 2 than the signed domination number in case of $n \equiv 1(\bmod 5)$, and $f$ remains a signed dominating function of the graph. Consequently:

$\gamma_{s}\left(P_{6} \times P_{n}\right)=2 n+2: n \equiv 2(\bmod 5) \quad$ (Figure 14$)$.

Case $n \equiv 0,3,4(\bmod 5)$.

In this case we delete the $B$ set vertices in the last column, then the signed domination number will increase by 4 than signed domination number in case of $n \equiv 1(\bmod 5)$. And $f$ remains a signed dominating function of the graph.

Consequently: $\gamma\left(P_{6} \times P_{n}\right)=2 n+4: n \equiv 0,3,4(\bmod 5)$ (Figure 15$)$.

\section{Lemma 2.1.}

Let $f$ be a signed domination function of $\left(P_{7} \times P_{n}\right)$, and $B$ the graph vertices set which having the weight -1 , Then for any $j$ were $1 \leq j \leq n-1$, then $\sum_{k=j}^{j+1}\left|B_{K}\right| \leq 5$. Except the following cases:

$(3, j),(5, j) \in B,(1, j),(3, j),(5, j) \in B,(2, j),(3, j),(5, j) \in B$ or $(3, j),(5, j),(7$, $j) \in B$. Then $\sum_{k=j}^{j+1}\left|B_{K}\right| \leq 6$ and in this case $\left|B_{j+2}\right|+\left|B_{j+3}\right| \leq 5$.

Proof:

For any $j$ were $1 \leq j \leq n$ then $\left|B_{j}\right| \leq 4$.

Case a. $\left|B_{j}\right|=4$ :

The $j+1^{\text {th }}$ column includes at most one of the $B$ set vertices, except case $(1, j)$, $(3, j),(5, j),(7, j) \in B$. then the $j+1^{\text {th }}$ column includes two of the $B$ set vertices (Figure 16).

Case b. $\left|B_{j}\right|=3$ :

The $j+1^{\text {th }}$ column includes at most two vertices except in the following cases:

$(1, j),(3, j),(5, j) \in B,(2, j),(4, j),(6, j) \in B,(3, j),(5, j),(7, j) \in B$. Then $\left|B_{j+1}\right|$ $=3$ (Figure 17).

Case c. $\left|B_{j}\right|=2$ :

The $j+1^{\text {th }}$ column includes at most three vertices, except in case $(3, j),(5, j) \in$ $B$, then the $j+1^{\text {th }}$ column includes four of the $B$ set vertices (Figure 18). 


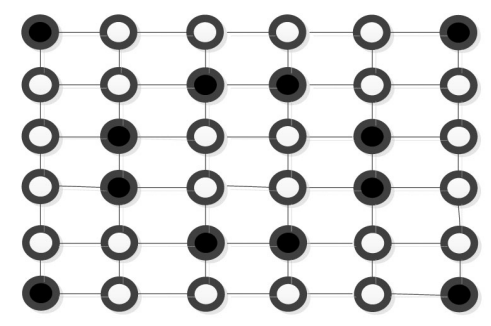

Figure 13. Case $n \equiv 1(\bmod 5)$.

$$
\begin{array}{lllllll}
0 & 0 & 0 & 0 & 0 & 0 & 0 \\
0 & 0 & 0 & 0 & 0 & 0 & 0 \\
0 & 0 & 0 & 0 & 0 & 0 & 0 \\
0 & 0 & 0 & 0 & 0 & 0 & 0 \\
0 & 0 & 0 & 0 & 0 & 0 & 0 \\
0 & 0 & 0 & 0 & 0 & 0 & 0
\end{array}
$$

Figure 14 . Case $n \equiv 2(\bmod 5)$.

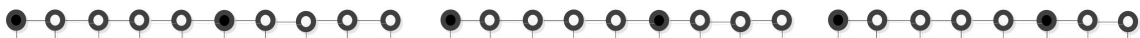

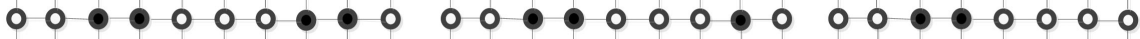

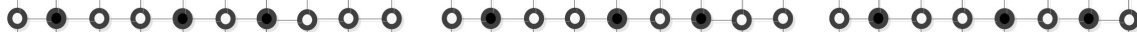

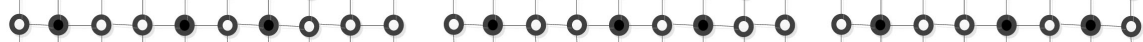

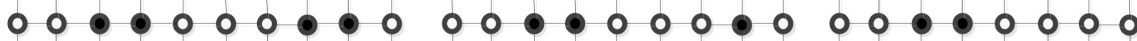

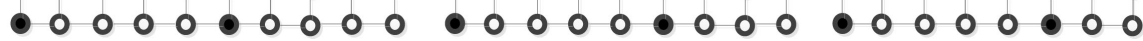

Figure 15. Case a.

$$
\begin{array}{lllllllllllllll}
0 & 0 & 0 & 0 & 0 & 0 & 0 & 0 & 0 & 0 & 0 & 0 & 0 & 0 & 0 \\
0 & 0 & 0 & 0 & 0 & 0 & 0 & 0 & 0 & 0 & 0 & 0 & 0 & 0 & 0 \\
0 & 0 & 0 & 0 & 0 & 0 & 0 & 0 & 0 & 0 & 0 & 0 & 0 & 0 & 0 \\
0 & 0 & 0 & 0 & 0 & 0 & 0 & 0 & 0 & 0 & 0 & 0 & 0 & 0 & 0 \\
0 & 0 & 0 & 0 & 0 & 0 & 0 & 0 & 0 & 0 & 0 & 0 & 0 & 0 & 0 \\
0 & 0 & 0 & 0 & 0 & 0 & 0 & 0 & 0 & 0 & 0 & 0 & 0 & 0 & 0 \\
0 & 0 & 0 & 0 & 0 & 0 & 0 & 0 & 0 & 0 & 0 & 0 & 0 & 0 & 0 \\
j-1 & j & j+1 & j-1 & j & j+1 & j-1 & j & j+1 & j-1 & j & j+1 & j-1 & j & j+1
\end{array}
$$

Figure 16. Case a.

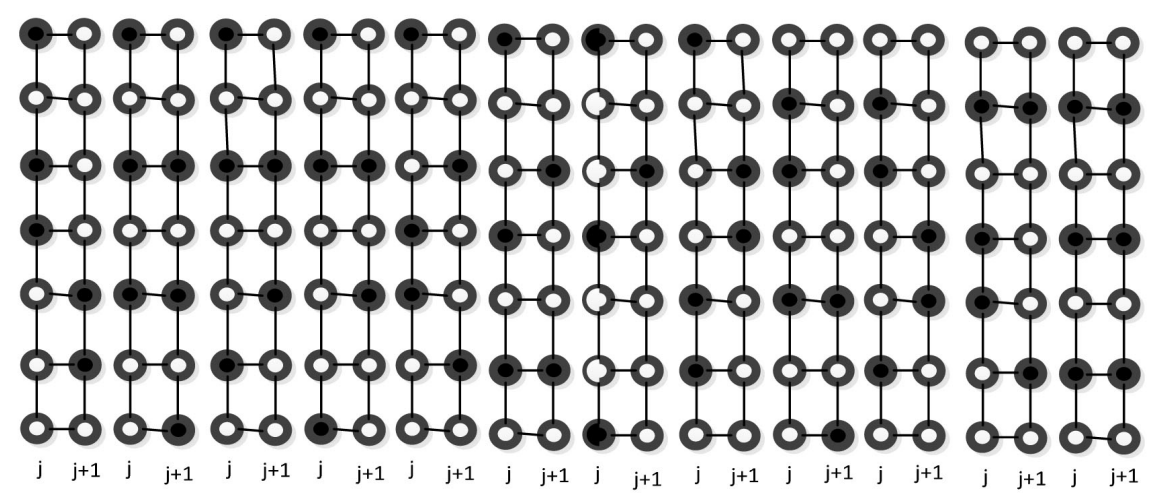

Figure 17. Case b. 


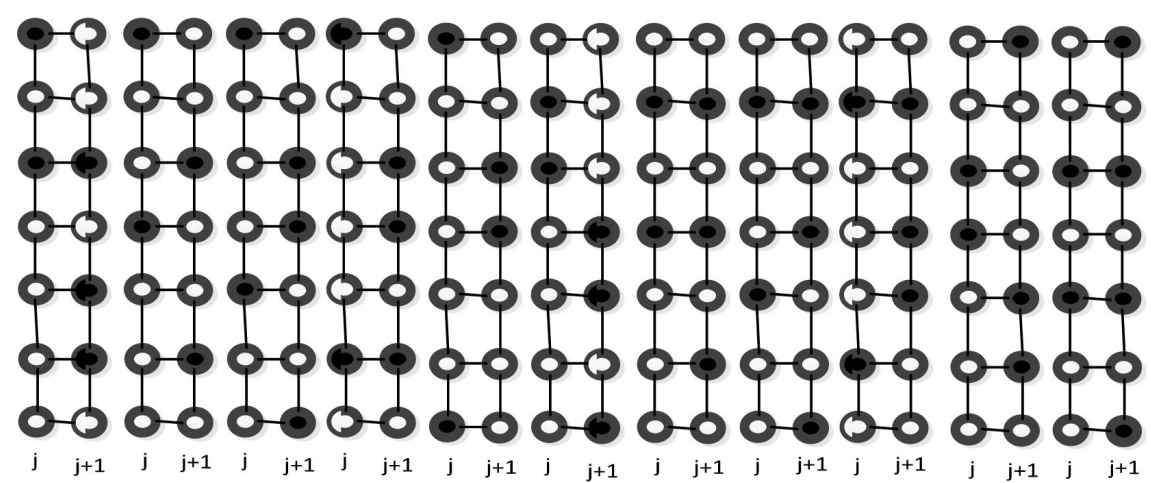

Figure 18. Case c.

In case $\left|B_{j}\right|=1$ or $\left|B_{j}\right|=0$ it's proofed easily because $\left|B_{j+1}\right| \leq 4$.

Lemma 2.2.

Let $f$ be a signed domination function of $\left(P_{7} \times P_{n}\right)$ and $B$ the graph vertices set which having the weight -1 , then $\left|B_{1}\right|+\left|B_{2}\right|+\left|B_{3}\right| \leq 6$. Except for a case $(2,3)$, $(3,3),(6,3) \in B$. Then $\left|B_{1}\right|+\left|B_{2}\right|+\left|B_{3}\right| \leq 7$. In this case $\left|B_{4}\right|=1$.

Proof:

Case a. $\left|B_{2}\right|=3$ :

If $(1,3),(3,3),(5,3) \in B$ or $(2,3),(4,3),(6,3) \in B$ then the second column include three vertices of the $B$ set vertices, and the first column doesn't include any one of the $B$ set vertices (Figure 19).

Case b. $\left|B_{2}\right|=2$ :

If $(1,3),(3,3),(7,3) \in B$ or $(1,3),(4,3),(5,3) \in B$ or $(1,3),(4,3),(6,3) \in B$, then the second column include two vertices of the $B$ set vertices, and the first column doesn't include any one of the $B$ set vertices.

If $(1,3),(3,3),(4,3) \in B$ or $(1,3),(3,3),(6,3) \in B$ or $(1,3),(5,3),(6,3) \in B$ or $(2,3),(3,3),(5,3) \in B$ or $(2,3),(4,3),(5,3) \in B$, then the second column include two vertices of the $B$ set vertices, and the first column include one of the $B$ set vertices.

If $(2,3),(3,3),(6,3) \in B$, then the second column include two vertices of the $B$ set vertices, and the first column include two vertices of the $B$ set vertices. In this case the fourth column at most include one of the $B$ set vertices (Figure 20).

Case b. $\left|B_{2}\right|=1$ :

If $(1,3),(4,3),(7,3) \in B$, then the second column include one of the $B$ set vertices, and the first column include one of the $B$ set vertices (Figure 21).

Remark 2.1. $\left|B_{n-2}\right|+\left|B_{n-1}\right|+\left|B_{n}\right| \leq 6$. Except for a case (2, $\left.n-2\right)$, (3, $\left.n-2\right)$, (6, $n-2) \in B$. Then $\left|B_{n-2}\right|+\left|B_{n-1}\right|+\left|B_{n}\right| \leq 7$. In this case $\left|B_{n-3}\right|=1$, and prove as in the lemma (2.2.)

Theorem 2.2. Let $n$ be a positive integer

If $n \equiv 0,2(\bmod 5)$, then $\gamma_{s}\left(P_{7} \times P_{n}\right)=\frac{11 n}{5}+6$;

If $n \equiv 1,3(\bmod 5)$, then $\gamma_{s}\left(P_{7} \times P_{n}\right)=\frac{11 n}{5}+7$; 


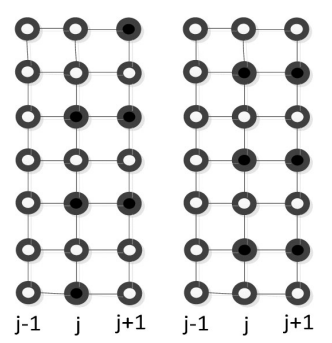

Figure 19. Case b.

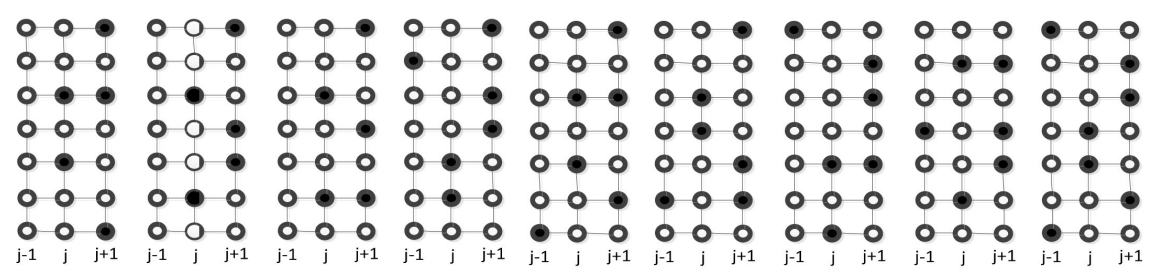

Figure 20. Case b.

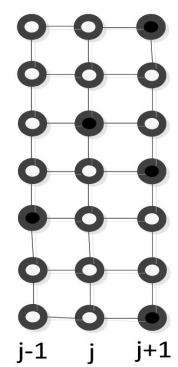

Figure 21. Case c.

If $n \equiv 4(\bmod 5)$, then $\gamma_{s}\left(P_{7} \times P_{n}\right)=\frac{11 n}{5}+8$.

Proof:

Case $n \equiv 0(\bmod 5)$.

Let $f$ be a signed domination function of the $P_{7} \times P_{n}$. And $B$ the graph vertices set which having the weight -1 . Then for any $j$ were $1 \leq j \leq n-3$ then $\sum_{k=j-1}^{j+3}\left|B_{K}\right| \leq 12$.

Case a. $\left|B_{j}\right|=4$ :

Then we discuss the following cases:

a-1. If $(2, j),(3, j),(5, j),(6, j) \in B$ then both of the $j-1^{\text {th }}, j+1^{\text {th }}$ columns don't include any one of the B set vertices, so $\left|B_{j-1}\right|+\left|B_{j}\right|+\left|B_{j+1}\right| \leq 4$. And according to lema1 then $\left|B_{j+2}\right|+\left|B_{j+3}\right| \leq 6$.

a-2. If $(1, j),(3, j),(4, j),(6, j) \in B$ or $(1, j),(3, j),(4, j),(7, j) \in \mathrm{B}$ or $(1, j),(3, j)$, $(5, j),(6, j) \in B$. Then one of the $j-1^{\text {th }}$ or $j+1^{\text {th }}$ column includes one of the B set vertices, as $\left|B_{j+2}\right|+\left|B_{j+3}\right| \leq 6$.

a-3. If $(1, j),(3, j),(5, j),(7, j) \in B$ then both of the $j-1^{\text {th }}, j+1^{\text {th }}$ columns include two of the $B$ set vertices, as $\left|B_{j+2}\right|+\left|B_{j+3}\right| \leq 6$ (Figure 22).

Case b. $\left|B_{j}\right|=3$ :

We discuss the following cases: 


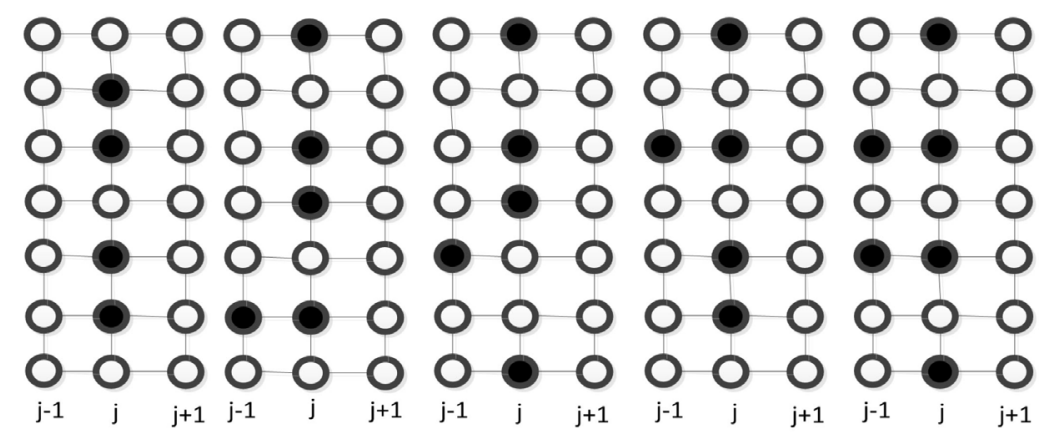

Figure 22. Case a.

b-1. If $(1, j),(4, j),(7, j) \in B$ then at most one of the $j-1^{\text {th }}$ columns vertices and also at most one of the $j+1^{\text {th }}$ vertices belongs to the $B$ set vertices. Then the number of the vertices from the $B$ set in the five successive columns remains less or equal to 12 (Figure 23 ).

b-2. If $(1, j),(3, j),(4, j) \in B$ or $(1, j),(4, j),(5, j) \in B$ or $(1, j),(4, j),(6, j) \in B$ or $(1, j),(5, j),(6, j) \in B$ or $(2, j),(3, j),(5, j) \in B$ or $(2, j),(3, j),(6, j) \in B$ or $(2$, $j),(4, j),(5, j) \in B$. then at most two of the $j-1^{\text {th }}$ columns vertices and also at most one of the $j+1^{\text {th }}$ vertices belongs to the $B$ set vertices. Then the number of the vertices from the $B$ set in the five successive columns remains less or equal to 12 (Figure 24).

b-3. If $(2, j),(4, j),(6, j) \in B$ then at most one of the two vertices $(2, j-1),(2, j$ $+1)$ and one of the two vertices $(4, j-1),(4, j+1)$, And one of the two vertices $(6, j-1),(6, j+1)$ may be of the $B$ set vertices. Then the number of the vertices from the $B$ set in the five successive columns remains less or equal to 12 (Figure 25).

b-4. If $(1, j),(3, j),(5, j) \in B$ then the $j-1^{\text {th }}$ column includes at most three of the B set vertices. In case $\left|B_{j-1}\right|=3$. Then $(3, j-1),(5, j-1),(7, j-1) \in B$. so $(6$, $j+1),(6, j+2) \in B$. Thus it remains in the $j+2^{\text {th }}$ column three successive vertices include at most two of the $B$ set vertices, so the $j+3^{\text {th }}$ column includes at most two of the $B$ set vertices (Figure 26).

b-5. If $(1, j),(3, j),(7, j) \in B$ then both of the $j-1^{\text {th }}, j+1^{\text {th }}$ columns include at most two of the $B$ set vertices.

b-5-1. If $(3, j-1),(5, j-1) \in B$ then $(4, j+1),(5, j+1) \in B$ and $(2, j+2),(6, j$ $+2) \in B$ then three of the $j+3^{\text {th }}$ column vertices belongs to the $B$ set vertices.

b-5-2. If $(4, j-1),(5, j-1) \in B$ then $(3, j+1),(5, j+1) \in B$, and $(2, j+2),(5$, $j+2) \in B$ or $(2, j+2),(6, j+2) \in B$, then at most three of the $j+3^{\text {th }}$ column vertices belong to the $B$ set vertices (Figure 27).

b-6. If $(1, j),(3, j),(6, j) \in B$ then the $j-1^{\text {th }}$ column includes at most two of the $B$ set vertices, in this case the $j+1^{\text {th }}$ column includes at most two of the $B$ set vertices, and the $j+2^{\text {th }}$ column includes at most three vertices and the $j+3^{\text {th }}$ column includes at most two vertices of the $B$ set vertices (Figure 28).

Case c. $\left|B_{j}\right|=2$ :

c-1. If $(1, j),(4, j) \in B$ or $(1, j),(7, j) \in B$ then both of the $j-1^{\text {th }}, j+1^{\text {th }}$ columns include at most two of the $B$ set vertices, then the $j-1^{\text {th }}, j^{\text {th }}, j+1^{\text {th }}$ columns 


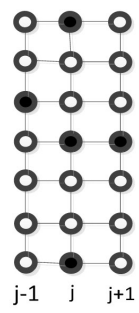

Figure 23. Case b-1.

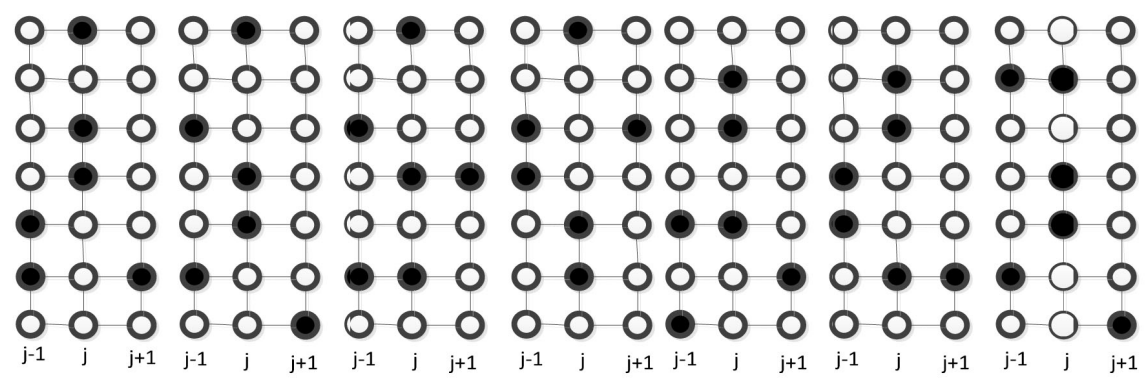

Figure 24. Case b-2.

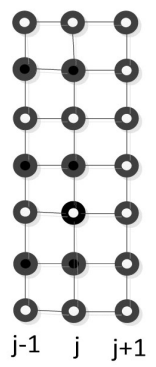

Figure 25. Case b-3.

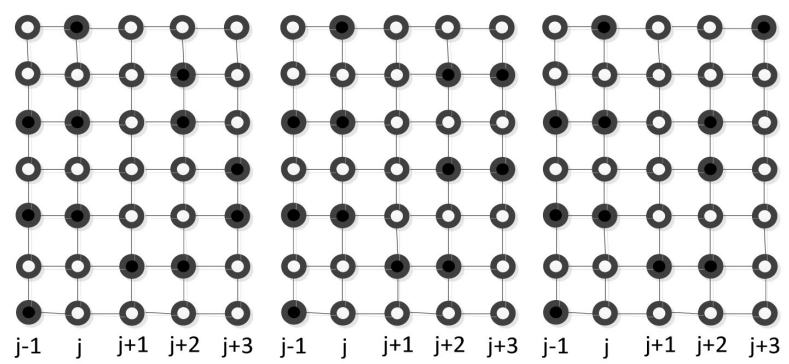

Figure 26. Case b-4.

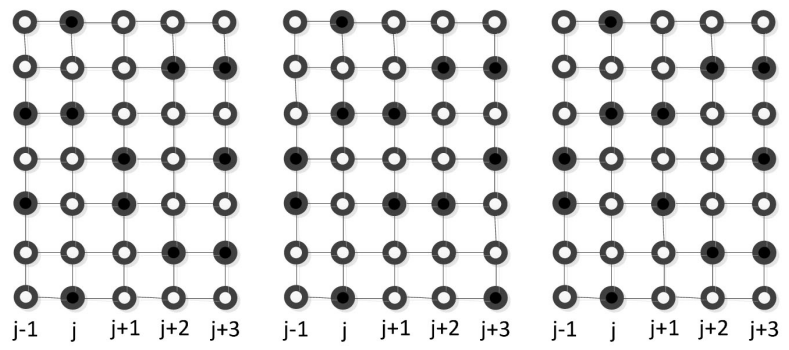

Figure 27. Case b-5. 


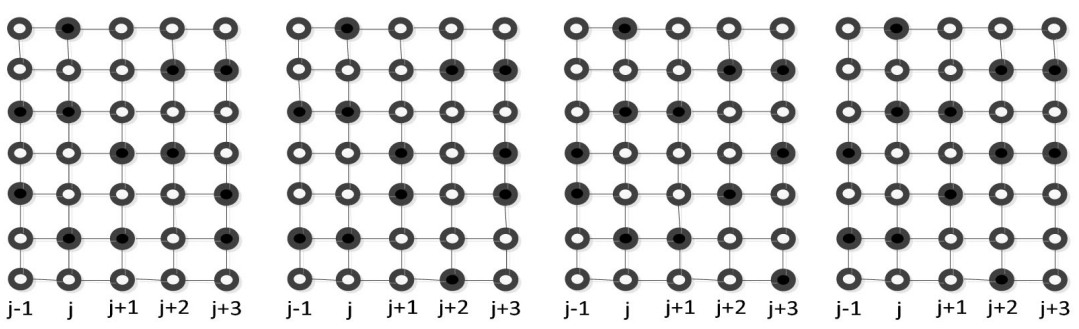

Figure 28. Case b-6.

include at most six of the $B$ set vertices, as any two columns include at most six vertices (Figure 29).

c-2. If $(1, j),(3, j) \in B$ then the $j-1^{\text {th }}$ column includes at most three vertices, because one of the two vertices $(3, j-1) \in B$ or $(4, j-1) \in B$ and either the two vertices $(5, j-1)$ and $(6, j-1)$ or $(5, j-1)$ and $(7, j-1)$ belong to the $B$ set vertices.

c-2-1. If $(3, j-1) \in B$ the $j+1^{\text {th }}$ column includes at most three of the $B$ set vertices, in this case the $j+2^{\text {th }}$ column includes at most one of the $B$ set vertices, and the $j+3^{\text {th }}$ column includes at most three vertices. Or the $j+2^{\text {th }}$ column includes two of the $B$ set vertices and the $j+3^{\text {th }}$ column includes at most three vertices.

c-2-2. If $(4, j-1) \in \mathrm{B}$ then the $j+1^{\text {th }}$ column includes at most three of the $B$ set vertices, in this case $(3, j+1),(5, j+1),(6, j+1) \in B$ and $(2, j+2) \in B$, so $(2$, $j+3),(4, j+3),(5, j+3),(7, j+3) \in B$, then the $j-2^{\text {th }}$ column includes at most one of the B set vertices, then $\sum_{k=j-2}^{j+2}\left|B_{K}\right| \leq 12$. Also the $j+4^{\text {th }}$ column doesn't include any one of the $B$ set vertices, so $\sum_{k=j}^{j+4}\left|B_{K}\right| \leq 12$. And according to lemma 2-1 note $\left|B_{j+5}\right|+\left|B_{j+6}\right| \leq 6$, so $\left|B_{j+7}\right| \leq 6$. Then every ten successive columns include at most twenty four of the $B$ set vertices (Figure 30 ).

c-3. If $(1, j),(5, j) \in B$ then the $j-1^{\text {th }}$ column includes at most three of the $B$ set vertices, so the $j+1^{\text {th }}$ and $j+2^{\text {th }}$ columns includes at most two of the $B$ set vertices, and the $j+3^{\text {th }}$ column includes at most three vertices (Figure 31 ).

c- 4 . If $(1, j),(6, j) \in B$ then the $j-1^{\text {th }}$ column includes at most three vertices, in this case the $j+1^{\text {th }}$ column includes at most two of the $B$ set vertices, also the $j$ $+2^{\text {th }}$ column includes three of the $B$ set vertices, and the $j+3^{\text {th }}$ column includes at most two vertices (Figure 32).

c-5. If $(2, j),(3, j) \in B$ then the $j-1^{\text {th }}$ column includes at most three of the $B$ set vertices, then the $j+1^{\text {th }}$ column includes two of the $B$ set vertices which are $(5, j+1),(6, j+1)$, also $(1, j+2),(3, j+2),(4, j+2) \in B$, and the $j+3^{\text {th }}$ column includes only one of the $B$ set vertices (Figure 33 ).

c-6. If $(2, j),(4, j) \in B$ then the $j-1^{\text {th }}$ column includes at most three of the $B$ set vertices, so the $j+1^{\text {th }}$ column includes at most two of the $B$ set vertices, in this case the $j+2^{\text {th }}$ column includes at most three of the $B$ set vertices, and the $j$ $+3^{\text {th }}$ column includes at most two vertices (Figure 34).

c-7. If $(2, j),(5, j) \in \mathrm{B}$ then the $j-1^{\text {th }}$ column includes at most three of the $B$ set vertices, and the $j+1^{\text {th }}$ column includes two of the $B$ set vertices, then the $j+$ $2^{\text {th }}, j+3^{\text {th }}$ columns include at most five of the $B$ set vertices (Figure 35 ). 


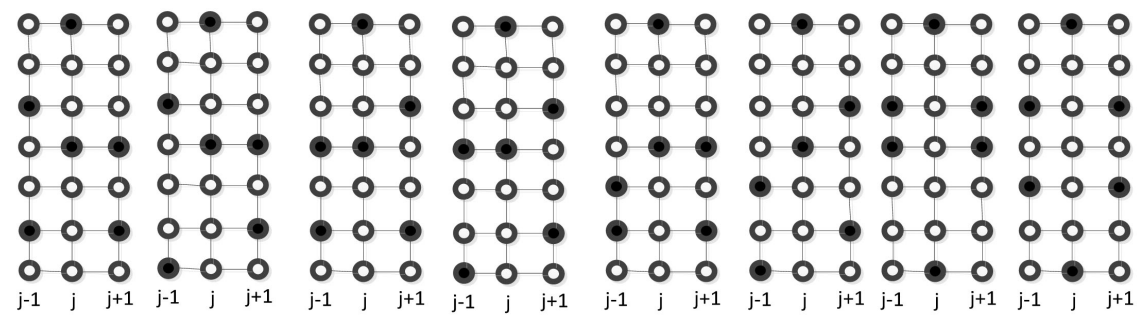

Figure 29. Case c-1.

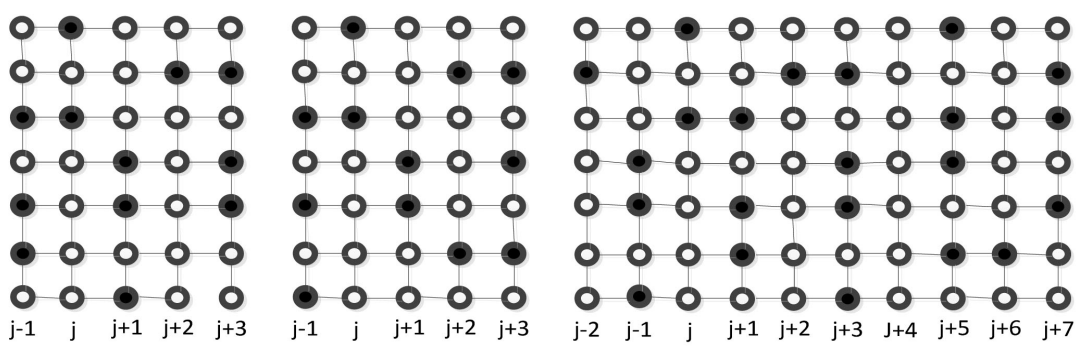

Figure 30. Case c-2.

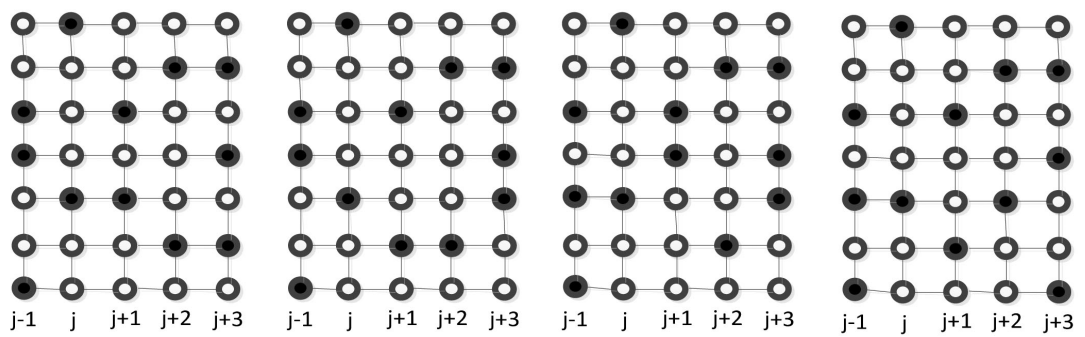

Figure 31. Case c-3.

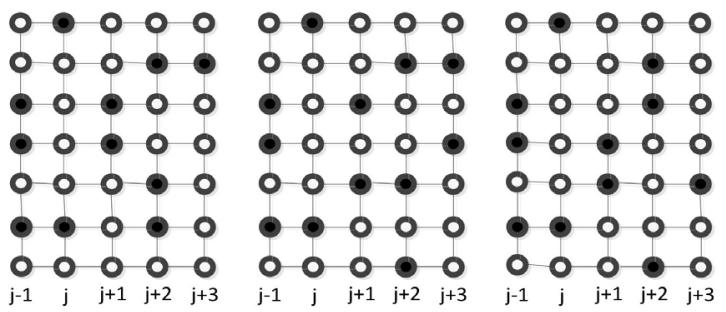

Figure 32. Case c-4.

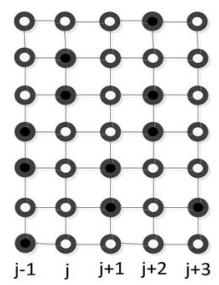

Figure 33. Case c-5.

c-8. If $(2, j),(6, j) \in \mathrm{B}$ then both of the $j-1^{\text {th }}, j+1^{\text {th }}$ columns include at most three of the $B$ set vertices, so the $j+2^{\text {th }}$ column includes at most one of the $B$ set vertices, and the $j+3^{\text {th }}$ column includes at most three vertices (Figure 36). 


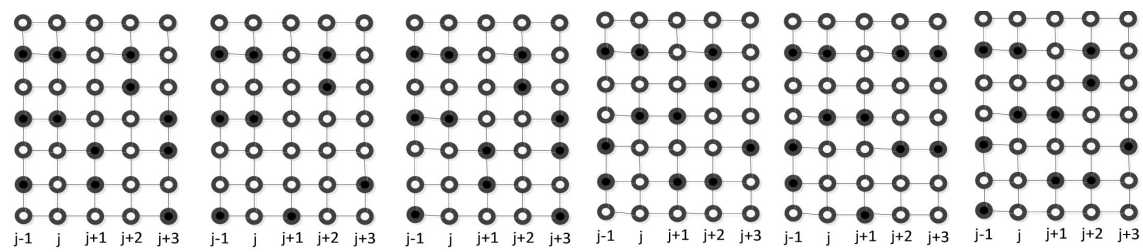

Figure 34. Case c-6.

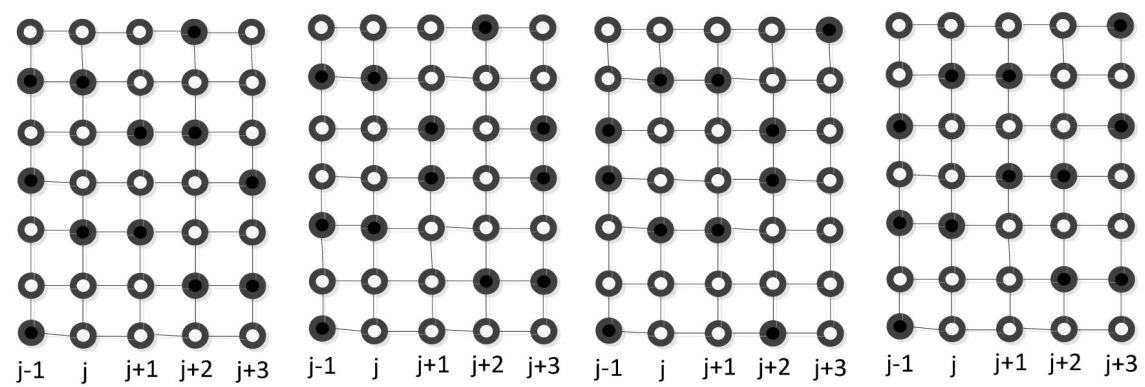

Figure 35. Case c-7.

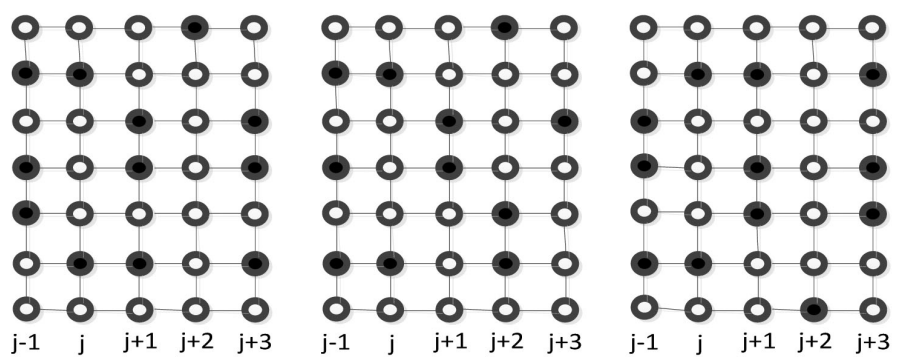

Figure 36. Case c-8.

c-9. If $(3, j),(4, j) \in \mathrm{B}$ then the $j-1^{\text {th }}$ column includes at most three of the $B$ set vertices, then the $j+1^{\text {th }}$ column includes at most three of the $B$ set vertices, then the $j+2^{\text {th }}$ column includes only one of the $B$ set vertices, and the $j+3^{\text {th }}$ column includes at most three vertices (Figure 37).

c-10. If $(3, j),(5, j) \in B$ then the $j-1^{\text {th }}$ column includes at most four of the $B$ set vertices, so the $j+1^{\text {th }}$ column includes at most two vertices, then the $j+2^{\text {th }}$ column includes at most three of the $B$ set vertices, and the $j+3^{\text {th }}$ column includes at most one vertex (Figure 38).

Case d. $\left|B_{j}\right|=1$ :

In this case the $j+1^{\text {th }}, j+2^{\text {th }}$ columns include at most five of the $B$ set vertices, so if the $j+3^{\text {th }}, j+4^{\text {th }}$ columns include six of the $B$ set vertices, then the number of the vertices in the five columns is less or equal to 12 (Figure 39 ).

We note from all the previous cases $|B| \leq \frac{12 n}{5}$. Then $\gamma_{s}\left(P_{7} \times P_{n}\right) \geq$ $7 n-2\left(\frac{12 n}{5}\right)=\frac{11 n}{5}$.

To find the upper bound of the signed domination number of $\left(P_{7} \times P_{n}\right)$ graph, let's define (Figure 40). 


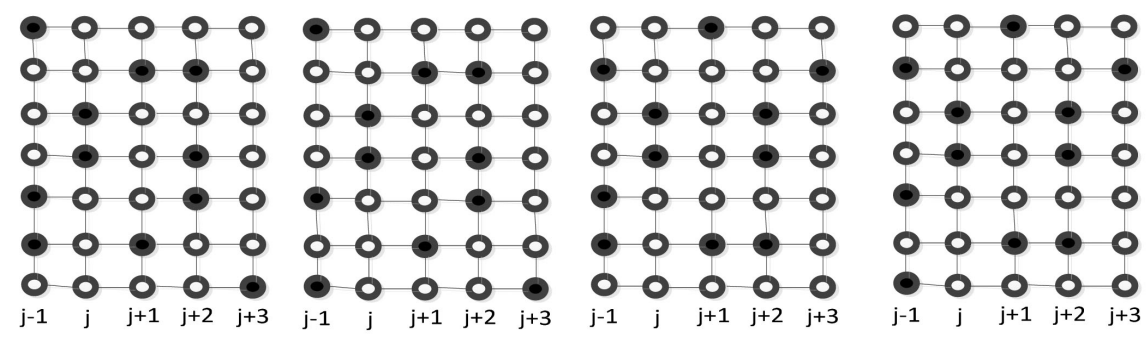

Figure 37. Case c-9.

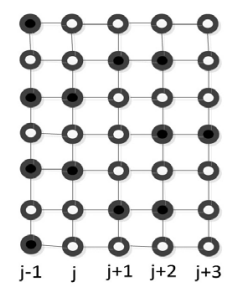

Figure 38. Case c-10.

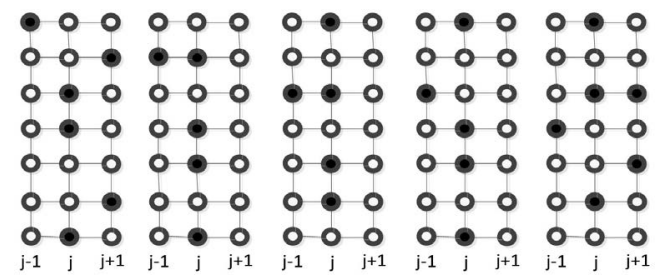

Figure 39. Case d.

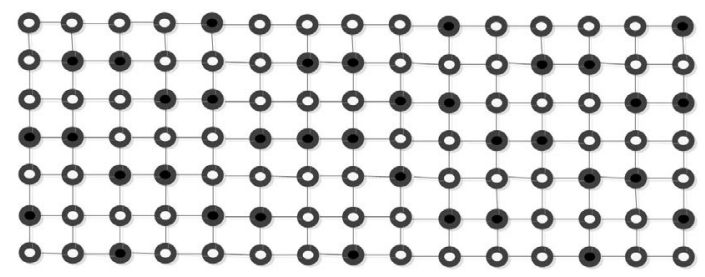

Figure 40. Case B.

$$
\begin{aligned}
B=\{ & (4,5 j),(6,5 j): 0 \leq j \leq\left\lfloor\frac{n}{5}\right\rfloor \\
& \cup(2,5 j+1),(4,5 j+1),(6,5 j+1): 0 \leq j \leq\left\lfloor\frac{n-1}{5}\right\rfloor \\
& \cup(2,5 j+2),(5,5 j+2),(7,5 j+2): 0 \leq j \leq\left\lfloor\frac{n-2}{5}\right\rfloor \\
& \cup(3,5 j+3),(5,5 j+3): 0 \leq j \leq\left\lfloor\frac{n-3}{5}\right\rfloor \\
& \left.\cup(1,5 j+4),(3,5 j+4),(6,5 j+4): 0 \leq j \leq\left\lfloor\frac{n-4}{5}\right\rfloor\right\}
\end{aligned}
$$

If $B$ the graph vertices set which having the weight -1 , then every one of the $P_{7}$ $\times P_{n}$ graph vertices achieves the signed domination function and $|B| \geq\left\lfloor\frac{12 n}{5}\right\rfloor$. 
According to lemma 2-2 we deleted the vertex $(4,1)$ from the previously defined set $B$ vertices in all cases, then $\gamma_{s}\left(P_{7} \times P_{n}\right) \geq\left\lfloor\frac{11 n}{5}\right\rfloor+2$.

Case $n \equiv 0,2(\bmod 5)$.

According to lemma $2-2$, then in case $n \equiv 0(\bmod 5)$, we delete the vertices (3, $n)$, $(6, n)$, so in case $n \equiv 2(\bmod 5)$, we delete the vertex $(4, n)$. Then the signed domination number will increase by 4 .

Consequently: $\quad \gamma_{s}\left(P_{7} \times P_{n}\right)=\left\lfloor\frac{11 n}{5}\right\rfloor+2+4=\left\lfloor\frac{11 n}{5}\right\rfloor+6: n \equiv 0,2(\bmod 5)$

(Figure 41).

Case $n \equiv 1,3(\bmod 5)$.

When we add one column on case $n \equiv 0(\bmod 5)$, note that the number of vertices will increase by 7 , and the number of set $B$ vertices will increase by 2 , in this case

$$
\gamma_{s}\left(P_{7} \times P_{n}\right)=\left\lfloor\frac{11(n-1)}{5}\right\rfloor+2+7=\left\lfloor\frac{11 n}{5}\right\rfloor+7: n \equiv 1(\bmod 5) .
$$

When we add three columns on case $n \equiv 0(\bmod 5)$, note that the number of vertices will increase by 21 , and the number of set $B$ vertices will increase by 5 , in this case

$$
\gamma_{s}\left(P_{7} \times P_{n}\right)=\left\lfloor\frac{11(n-3)}{5}\right\rfloor+2+21-2 \times 5=\left\lfloor\frac{11 n}{5}\right\rfloor+7: n \equiv 3(\bmod 5) .
$$

Consequently: $\gamma_{s}\left(P_{7} \times P_{n}\right) \geq\left\lfloor\frac{11 n}{5}\right\rfloor+7: n \equiv 1,3(\bmod 5)$. (Figure 42)

Case $n \equiv 4(\bmod 5)$.

When we add four column on case $n \equiv 0(\bmod 5)$, note that the number of vertices will increase by 28 , and the number of set $B$ vertices will increase by 9 , in this case (Figure 43)

$$
\begin{array}{lll|l|l|l}
\gamma_{s}\left(P_{7} \times P_{n}\right)=\left\lfloor\frac{11(n-4)}{5}\right\rfloor+2+28-2 \times 7=\left\lfloor\frac{11 n}{5}\right\rfloor+8: n \equiv 4(\bmod 5) . \\
0
\end{array}
$$

Figure 41. Case $n \equiv 0,2$ (mode 5$)$.

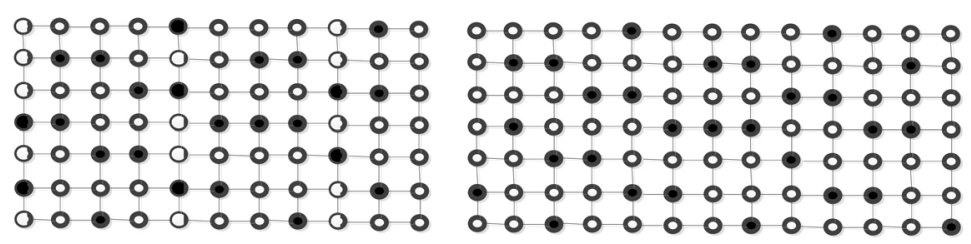

Figure 42 . Case $n \equiv 1,3$ (mode 5$)$. 


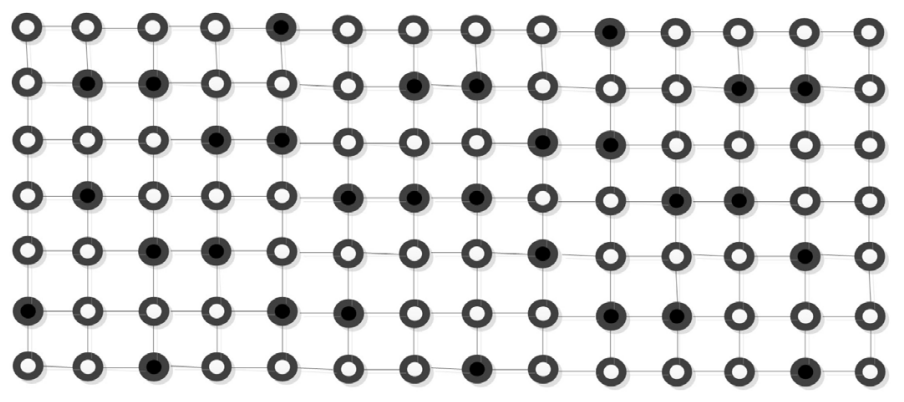

Figure 43. Case $n \equiv 4$ (mode 5$)$.

\section{Conclusion}

In this paper, we studied the signed domination numbers of the Cartesian product of two paths $P_{m}$ and $P_{n}$ for $m=6,7$ and arbitrary $n$. We will work to find the signed domination numbers of the Cartesian product of two paths $P_{m}$ and $P_{n}$ for arbitraries $m$ and $n$, and special graphs.

\section{Conflicts of Interest}

The authors declare no conflicts of interest regarding the publication of this paper.

\section{References}

[1] Dunbar, J., Hedetniemi, S.T., Henning, M.A. and Slater, P.J. (1995) Signed Domination in Graph Theory. In: Combinatorics and Applications, Wiley, New York, 1, 311-322.

[2] Broere, I., Hattingh, J.H., Henning, M.A. and McRae, A. (1995) Majority Domination in Graphs. Discrete Mathematics, 138, 125-135.

https://doi.org/10.1016/0012-365X(94)00194-N

[3] Cockayne, E.J. and Mynhardt, C.M. (1996) On a Generalization of Signed Dominating Functions of Graphs. Ars Combinatoria, 43, 235-245.

[4] Favaron, O. (1995) Signed Domination in Regular Graphs. Discrete Mathematics, 158, 287-293. https://doi.org/10.1016/0012-365X(96)00026-X

[5] Haasa, R. and Wexlerb, T.B. (2004) Signed Domination Numbers of a Graph and Its Complement. Discrete Mathematics, 283, 87-92. https://doi.org/10.1016/j.disc.2004.01.007

[6] Hosseini, S.M. (2015) New Bounds on the Signed Domination Numbers of Graphs. Australasian Journal of Combinatorics, 61, 273-280.

[7] Hassan, M., AL Hassan, M. and Mostafa, M. (2020) The Signed Domination Number of Cartesian Product of Two Paths. Open Journal of Discrete Mathematics, 10, 45-55. https://doi.org/10.4236/ojdm.2020.102005 